\title{
Absorción Acústica de Jardines Verticales
}

\author{
Acoustic Absorption of Vertical Gardens \\ Ariel Velis ${ }^{\dagger 1}$, Carlos Posse ${ }^{\dagger 1}$, Ana Rizzo La Malfa ${ }^{* 2}$ \\ ${ }^{\dagger}$ Laboratorio de Acústica y Luminotecnia de la CIC. \\ Cno. Centenario y 506, J. M. Gonnet, Buenos Aires, Argentina \\ ${ }^{1}$ ciclalegba.gob.ar \\ * Doctoranda Facultad de Arquitectura y Urbanismo, UNLP \\ ${ }^{2}$ rizzo. lamalfa@gmail.com \\ Recibido: 19/10/18; Aceptado: 06/02/19
}

\begin{abstract}
Vertical gardens, called "Greenery Systems", are a new tendency in modern constructions. They are applied increasingly more frequently on those buildings where energy and ecological considerations, environmentally friendly, are a priority. Some main features of these technologies are: thermal insulation improvement, environmental pollution filtering, acoustic absorption, water retention and a friendly aesthetic appearance. Vertical gardens can have dissimilar acoustic features because they depend on the kind of support, substrate and vegetation used. For this reason, nowadays, collected data are very diverse and incomplete and it is necessary to expand the data available to provide more alternatives with practical application. In this work, acoustic absorption of modular vertical greening was studied. Each module was developed with a rigid PVC base covered with fibrous material forming pockets, where the substrate and vegetation were placed. The plant species that were used are some of the most widespread ones in the region, in order to achieve a good adaptation and simple maintenance.
\end{abstract}

Keywords: vertical greenery systems; acoustic absorption; noise reduction.

Resumen - Los jardines verticales forman parte de una de las tendencias tecnológicas constructivas más modernas, conocidas como "Greenery Systems". Estas son aplicadas cada vez con más frecuencia en aquellas construcciones donde se priorizan consideraciones energéticas y ecológicas conciliables con el medio ambiente. Algunas de las características relevantes que presentan son la mejora del aislamiento térmico, el filtrado de polución ambiental, la absorción acústica, la retención de agua y una estética amigable. Los jardines verticales pueden poseer características acústicas disímiles entre ellos, debido a que dependen del tipo de soporte, sustrato y vegetación utilizada. Por ese motivo en la actualidad los datos existentes son muy variados e incompletos, y resulta necesario ampliar los mismos para disponer de mayores variantes constructivas con aplicación práctica. En este trabajo se estudió la absorción acústica de un sistema de jardín vertical del tipo modular. Cada módulo fue realizado sobre una base rígida de PVC, revestida de material fibroso, con el cual se conformaron bolsillos. En ellos se alojó el sustrato y la vegetación. Las especies vegetales que se utilizaron son algunas de las más difundidas en la región, a fin de lograr una buena adaptación y un mantenimiento sencillo.

Palabras clave: jardines verticales; absorción acústica; reducción de ruido; greenery.

\section{INTRODUCCIÓN}

En las últimas décadas surge una situación extraordinaria en la historia de la humanidad como consecuencia del incremento significativo de la población mundial residente en áreas urbanas, situación que configura una matriz compleja de problemáticas tanto en el ambiente natural como en el construido. Dicha situación produce un conjunto de consecuencias en el aspecto sanitario, urbano y ambiental, entre las cuales se encuentra la contaminación acústica. En contrapartida, el desarrollo de "Greenery Systems" ha demostrado ser una solución apropiada para éstas problemáticas urbanas ya que, paulatinamente, se van reduciendo los costos de producción, mantenimiento, facilidad de colocación y además buscando cubrir las necesidades funcionales que requieren las viviendas en la actualidad. Los jardines verticales se presentan en diversos formatos ya sea en forma de cobertura intensiva o extensiva, parcial o total, compacta o modular, cada una de las cuales posee ventajas y desventajas respecto a los muros que se desea intervenir. La composición del jardín se conforma básicamente por una estructura que relaciona físicamente el muro con el sistema que contiene el sustrato, la vegetación y el sistema de riego.

\section{SISTEMA ESTUDIADO}

\section{A. Módulos individuales}

El tipo de jardín vertical que se estudia es el "modular". Este jardín vertical, en su versión completa y con aplicación práctica, está compuesto por paneles individuales (módulos) 
con dimensiones de $1,2 \mathrm{~m} \times 0,8 \mathrm{~m}\left(0,96 \mathrm{~m}^{2}\right)$. Cada uno de ellos está conformado por una placa de PVC espumado revestida en fieltro tramado de fibras de algodón sobre el cual, y con el mismo material, se ubican 25 bolsillos (5 filas de 5 bolsillos) para alojar el sustrato y los plantines. La Figura 1 muestra un esquema constructivo de cada módulo.
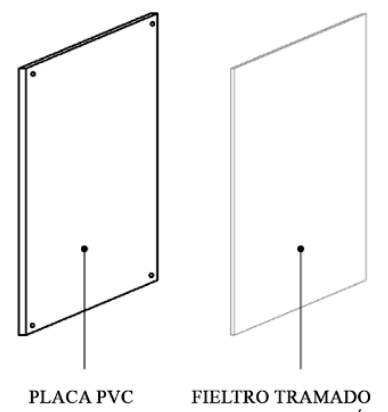
ESPUMADO FIELTRO TRAMADO
FIBRAS DE ALGODÓN E: $10 \mathrm{MM}$ E. $5 \mathrm{MM}$ FIETTRO TRAMADO DENVAS

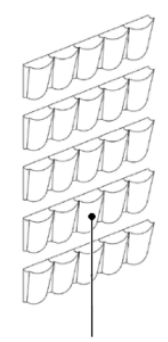

BOLSILLOS FIBRAS DE ALGODÓN CORRESP. A ESPECIE E: 5 MM

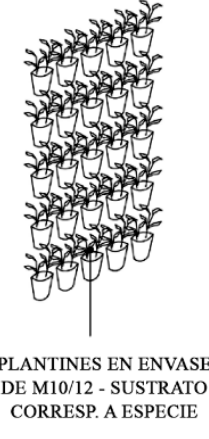

Fig. 1. Esquema de un módulo del jardín vertical estudiado

Los módulos fueron desarrollados y provistos por el Laboratorio Bio-Ambiental de Diseño de la Facultad de Planeamiento Socio-AmbientalArquitectura de la Universidad de Flores. La Figura 2 muestra fotografías de los detalles del sistema de soporte (placas PVC y fieltro).
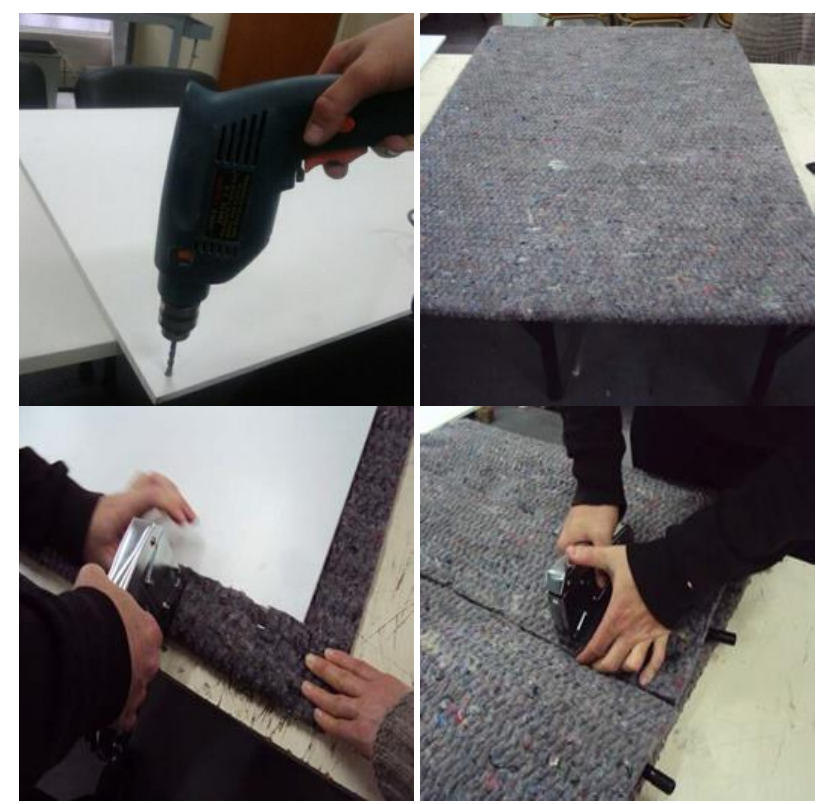

Fig. 2. Detalle de la construcción de los paneles.

Este tipo de jardín vertical se construye revistiendo el muro a tratar con el número necesario de módulos adyacentes montados sobre una estructura fijada a dicho muro. La misma está formada por una grilla de montantes y soleras que deja un espacio de aire intermedio de unos $5 \mathrm{~cm}$ de espesor. El sistema completo cuenta también con un sistema de riego por goteo que provee nutrientes y humedad a las plantas.

\section{B. Muestra representative de medición}

Para las mediciones en laboratorio, se utilizó como muestra representativa del sistema, un jardín formado por una estructura de listones de madera $(0,05 \mathrm{~m} \times 0,076 \mathrm{~m})$ sobre la cual se montaron 12 módulos de acuerdo al esquema de la Figura 3. Se conformó así una superficie total de $11,53 \mathrm{~m}^{2}$ lo cual satisface los requerimientos de la norma aplicada para la medición.

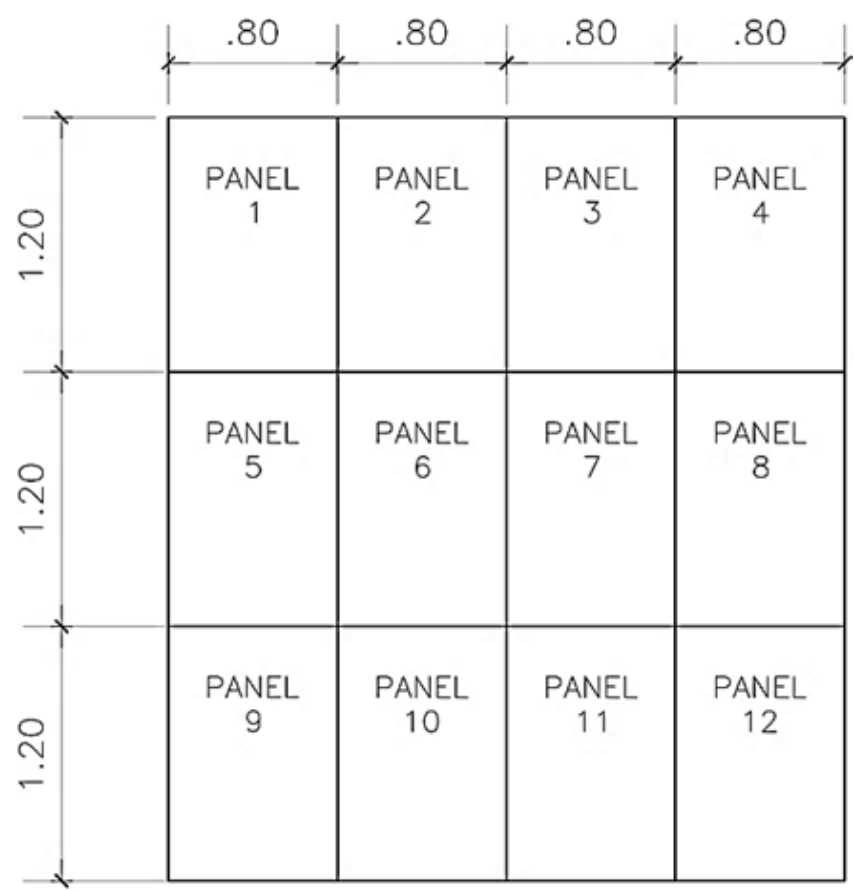

Fig. 3. Esquema de armado de paneles para ensayo de absorción.

La Figura 4 muestra el detalle y dimensiones de frente y perfil. Por cuestiones prácticas, y debido a que el sistema se pretendía montar y desmontar en diferentes escenarios para la medición, se descartó el sistema de riego en la muestra de medición.

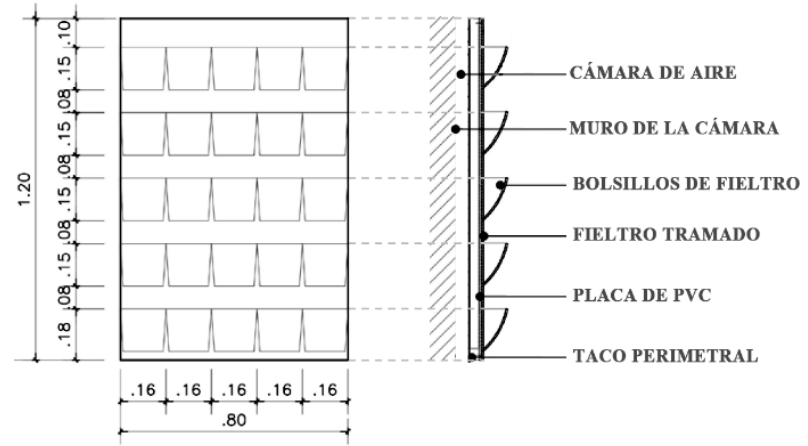

Fig. 4. Detalle vista y corte de paneles para ensayo de absorción 
III. PRIMERA ETAPA: MEDICIÓN DE LA ABSORCIÓN ACÚSTICA DEL SISTEMA SIN SUSTRATO NI PLANTAS

Los 12 paneles modulares fueron organizados en tres filas y cuatro columnas dentro de la cámara reverberante del Laboratorio de Acústica y Luminotecnia de la C.I.C. (L.A.L.). Estos fueron sujetados a la estructura de madera que estaba fija a una de las paredes de la cámara, empleando tornillos auto-perforantes. Los listones de los extremos ofrecían aislamiento perimetral ante la posible filtración de energía sonora hacia el interior de la cavidad de aire de $0,05 \mathrm{~m}$ de espesor entre cada panel y el muro de la cámara. De esta manera se pudo recrear una condición similar a la realidad, estando los paneles en forma vertical y con una cámara de aire similar a la que daría la estructura definitiva en la práctica. En esta etapa de medición fueron excluidos el sustrato y los plantines ya que se desea conocer el comportamiento absorbente de la base. En la Figura 5 se puede ver el montaje del sistema en la cámara reverberante y en la Figura 6 los detalles de la muestra ensayada en esta etapa.

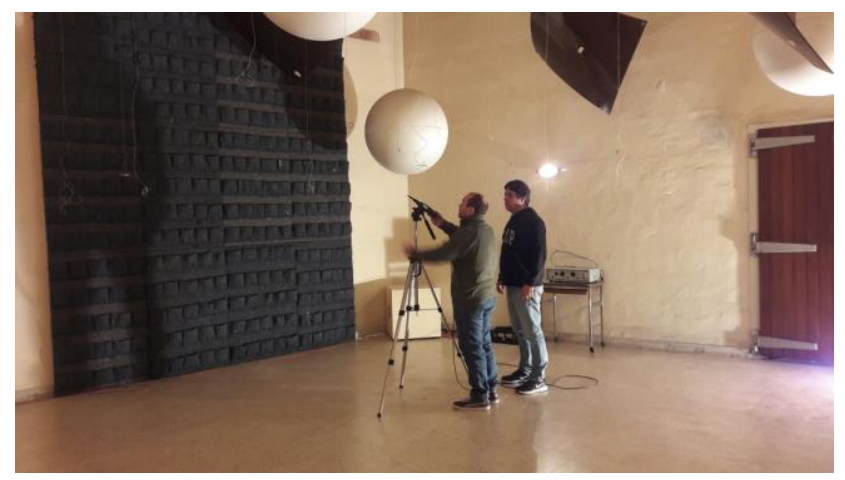

Fig. 5. Montaje y medición de la muestra en la cámara reverberante.
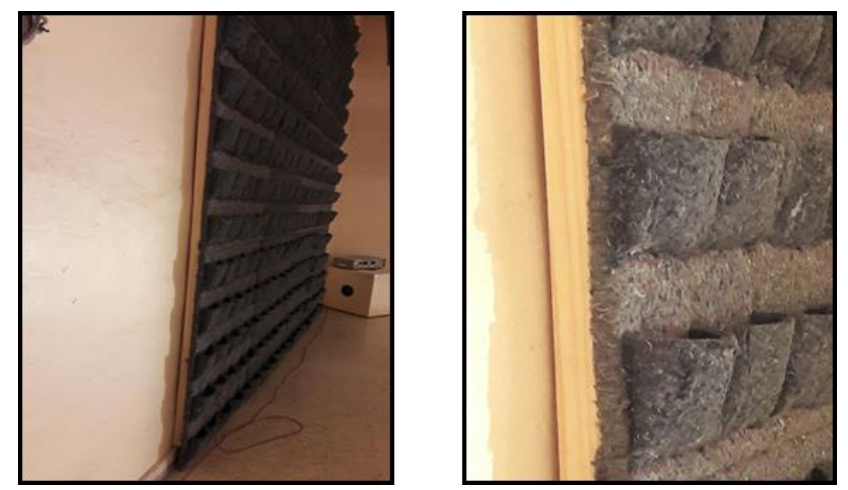

Fig. 6. Detalles de la muestra ensayada.

\section{A. Metodología de las Mediciones}

Para calcular la absorción sonora se midieron tiempos de reverberación siguiendo el procedimiento de la Norma ISO 354/1985 [3]. La sala reverberante utilizada posee un volumen de 189 $\mathrm{m}^{3}$, una superficie interior de $208 \mathrm{~m}^{2}$, y cumple con los requisitos de esta norma. Durante la medición se utilizaron 2 posiciones diferentes de las fuentes sonoras y 6 posiciones del micrófono. Se realizaron 3 registros por cada combinación fuente-micrófono, con lo cual, cada tiempo de reverberación fue el resultado del promedio de 36 caídas, siguiendo los recaudos expuestos en la norma antes citada.

Se calculó el coeficiente de absorción sonora $\alpha$ (adimensional) para las bandas de tercios de octava comprendidas entre 100 y $5000 \mathrm{~Hz}$. Además, se obtuvo el valor del Noise Reduction Coefficient (NRC), y el valor del Sound Absorption Average (SAA), de acuerdo con lo establecido en la norma ASTM C423-02a [4]:

- Noise Reduction Coefficient (NRC): obtenido como el promedio de los coeficientes de absorción sonora de las bandas de tercios de octavas centradas en 250, 500, 1000 y $2000 \mathrm{~Hz}$, redondeado al múltiplo más próximo de 0,05 .

- Sound Absorption Average (SAA): obtenido como el promedio de los coeficientes de absorción sonora de las bandas de tercios de octava comprendidas entre 200 y $2500 \mathrm{~Hz}$, redondeado al múltiplo más próximo de 0,01 .

\section{SEGUNDA ETAPA: COLOCACIÓN DE SUSTRATO Y LOS PLANTINES. DESARROLLO DE LOS MISMOS}

Posteriormente a la medición del sistema sin vegetación, este se completó colocando el sustrato y los plantines iniciándose de esta manera la etapa de desarrollo. Para esto se realizaron tareas de mantenimiento y riego en el predio del Laboratorio de Acústica durante 11 meses.

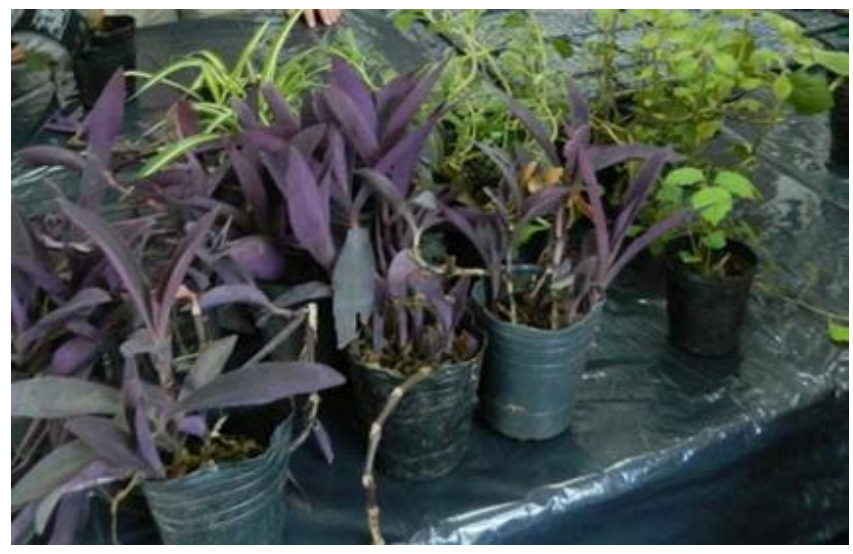

Fig. 7. Ejemplos de plantines utilizados 


\section{A. Sustrato}

Se utilizó un sustrato conformado por un $45 \%$ de materia orgánica y un $55 \%$ de cenizas, con un PH de entre 7,2 y 7,5 y una porosidad total de $72 \%$.

\section{B. Plantación y crecimiento}

Inicialmente se plantaron 4 especies de plantas: Incienso (Plectranthus coleoides), Plectranthus (Plectranthus neochilus), Tradescantia (Tradescantia pallida) y Salvia (Salvia procurrens). Todas ellas con una proporción similar en toda la muestra. En el transcurso de la etapa se destacó el crecimiento de algunas especies por sobre otras, por lo que se fueron reemplazando las especies más débiles por otras más fuertes y agregando nuevas.

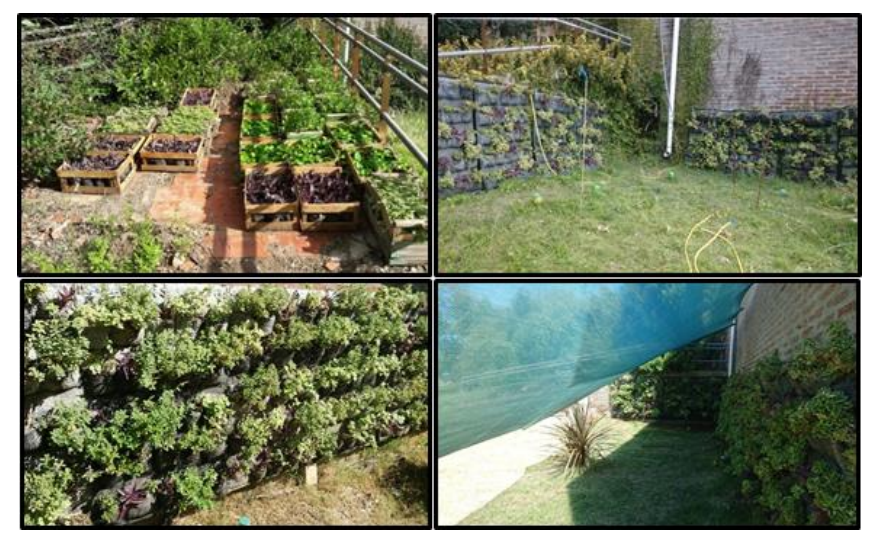

Fig. 8. Fotografías del período de crecimiento.

En las Figuras 7 y 8 se aprecian imágenes de la etapa de crecimiento.

Transcurrido el tiempo de desarrollo se procedió con la determinación de la absorción del sistema con las plantas en cámara reverberante. En ese momento, las especies vegetales presentes y sus proporciones fueron: Incienso (Plectranthus coleoides) 33\%, Plectranthus (Plectranthus neochilus) 40\%, Tradescantia (Tradescantia pallida) 25\%, Zebrina (Tradescantia Zebrina) 1\% y Manto de Virgen $1 \%$.

V. TERCER ETAPA: DETERMINACIÓN DE LA ABSORCIÓN SONORA EN CÁMARA REVERBERANTE DEL SISTEMA COMPLETO

\section{A. Metodología de las mediciones}

Para calcular la absorción acústica de la muestra con plantas se utilizó el mismo procedimiento utilizado para los paneles vacíos. Sin embargo, la operatoria se repitió para 3 mediciones que se corresponden con 3 condiciones de humedad del sistema (esto dependía de la cantidad de agua contenida tanto en el sustrato como en el fieltro de cada panel).

Se definieron entonces las 3 condiciones de humedad de la muestra, según se indica en la tabla I.

TABLA I

CONDICIONES DE HUMEDAD DE LA MUESTRA

\begin{tabular}{|c|c|}
\hline Condición de Humedad & Peso de cada panel \\
\hline A & $21,3 \mathrm{~kg}$ \\
\hline B & $22,8 \mathrm{~kg}$ \\
\hline C & $25,2 \mathrm{~kg}$ \\
\hline
\end{tabular}

Las mediciones se llevaron a cabo durante dos días consecutivos. En el primer día, y debido a que las plantas debían sufrir el menor estrés posible, se comenzó a trabajar a la hora 8 a.m. con el desmontaje de los paneles que estaban en la zona de crecimiento (predio exterior del LAL), en simultaneo con la medición de los tiempos de reverberación de la cámara reverberante vacía.

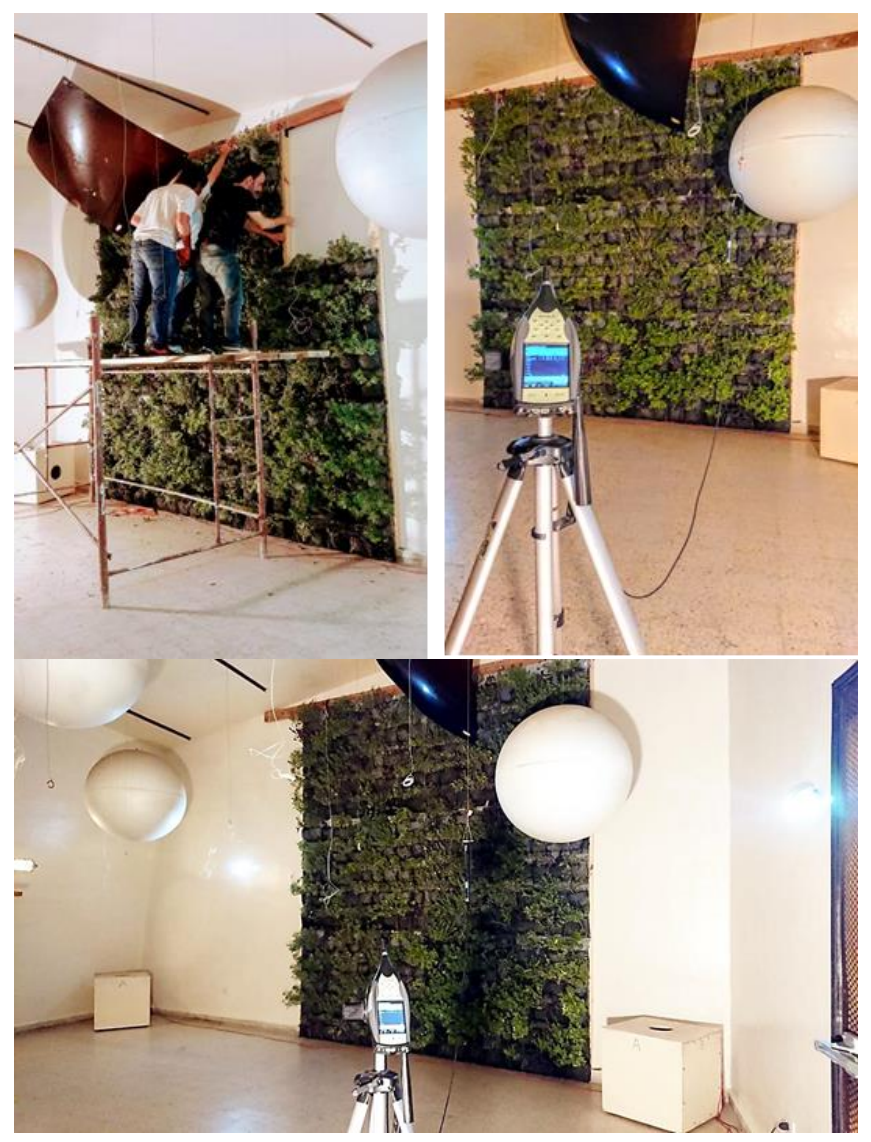

Fig. 9. Colocación y medición del sistema de jardín vertical completo.

Inmediatamente después se llevó a cabo el montaje de los paneles dentro de la cámara y a la 
hora 10 a.m. realizó la medición con la condición de humedad B. La muestra se mantuvo dentro de la cámara durante 24 horas sin recibir riego alguno. A la hora 10 a.m. del segundo día de medición se procedió a realizar la medición con la condición de humedad A. Inmediatamente luego de esta medición, se procedió al riego de la muestra, configurando la condición de humedad C y se midió en estas condiciones. Inmediatamente, se retiró la muestra y se midió nuevamente la sala vacía para permitir el cálculo de la absorción acústica de las últimas 2 mediciones. La Figura 9 muestra fotografías del montaje del sistema completo en la cámara reverberante del L.A.L.

\section{RESULTADOS OBTENIDOS}

En base a las mediciones realizadas, los valores calculados del coeficiente de absorción sonora del sistema en función de la frecuencia " $\alpha_{S}$ " (adimensional), se presentan en la Tabla II. Al final de la misma se expresan también los números únicos calculados NRC y SAA, de acuerdo con lo especificado en la norma ASTM C 423-02a.

TABLA II

COEFICIENTE DE ABSORCIÓN $\alpha$, NRC Y SAA

\begin{tabular}{|c|c|c|c|c|}
\hline \multirow{2}{*}{$\begin{array}{c}\text { Frecuencia } \\
{[\text { Hz] }}\end{array}$} & Paneles & \multicolumn{3}{|c|}{ Paneles con Sustrato y Plantas } \\
\hline & Vacíos & $\begin{array}{c}\text { Humedad } \\
\text { BAJA }\end{array}$ & $\begin{array}{c}\text { Humedad } \\
\text { MEDIA }\end{array}$ & $\begin{array}{c}\text { Humedad } \\
\text { ALTA }\end{array}$ \\
\hline $\mathbf{1 0 0}$ & 0,13 & 0,14 & 0,16 & 0,16 \\
\hline $\mathbf{1 2 5}$ & 0,35 & 0,29 & 0,28 & 0,29 \\
\hline $\mathbf{1 6 0}$ & 0,43 & 0,32 & 0,34 & 0,41 \\
\hline $\mathbf{2 0 0}$ & 0,48 & 0,49 & 0,53 & 0,46 \\
\hline $\mathbf{2 5 0}$ & 0,48 & 0,53 & 0,51 & 0,47 \\
\hline $\mathbf{3 1 5}$ & 0,53 & 0,49 & 0,4 & 0,48 \\
\hline $\mathbf{4 0 0}$ & 0,55 & 0,54 & 0,53 & 0,47 \\
\hline $\mathbf{5 0 0}$ & 0,59 & 0,53 & 0,54 & 0,49 \\
\hline $\mathbf{6 3 0}$ & 0,63 & 0,57 & 0,58 & 0,57 \\
\hline $\mathbf{8 0 0}$ & 0,70 & 0,67 & 0,67 & 0,66 \\
\hline $\mathbf{1 0 0 0}$ & 0,74 & 0,71 & 0,71 & 0,73 \\
\hline $\mathbf{1 2 5 0}$ & 0,79 & 0,76 & 0,82 & 0,78 \\
\hline $\mathbf{1 6 0 0}$ & 0,80 & 0,83 & 0,84 & 0,81 \\
\hline $\mathbf{2 0 0 0}$ & 0,84 & 0,87 & 0,87 & 0,86 \\
\hline $\mathbf{2 5 0 0}$ & 0,87 & 0,91 & 0,92 & 0,92 \\
\hline $\mathbf{3 1 5 0}$ & 0,92 & 0,97 & 0,95 & 0,93 \\
\hline $\mathbf{4 0 0 0}$ & 0,97 & 0,97 & 0,95 & 0,96 \\
\hline $\mathbf{5 0 0 0}$ & 1,02 & 0,99 & 0,95 & 0,97 \\
\hline $\mathbf{N R C}$ & 0,65 & 0,65 & 0,65 & 0,65 \\
\hline $\mathbf{S A A}$ & 0,67 & 0,66 & 0,67 & 0,64 \\
\hline & & & & \\
\hline
\end{tabular}

En la Figura 10 se observa un gráfico que muestra el coeficiente de absorción " $\alpha_{s}$ " tanto para los paneles vacíos (sin sustrato ni plantas) como para el sistema completo y con las 3 condiciones de humedad.

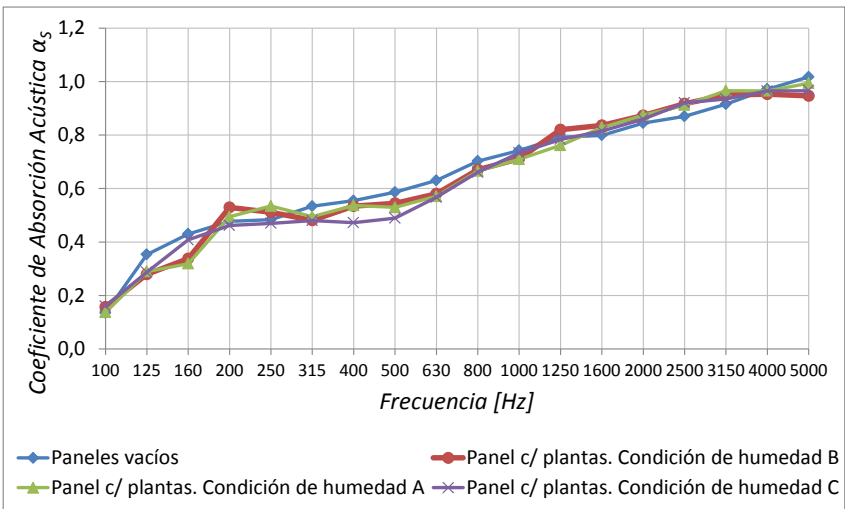

Fig. 10. Comparación de la absorción acústica de los paneles vacíos y con plantas con distintas condiciones de humedad.

El sistema ensayado se comparó con el comportamiento de los sistemas de las referencias 1 y 2 , juntamente con la absorción acústica de una pared desnuda (hormigón pintado). En la Figura 11 puede observarse esta comparación, en donde se ha tomado el panel ensayado con la condición de humedad B.

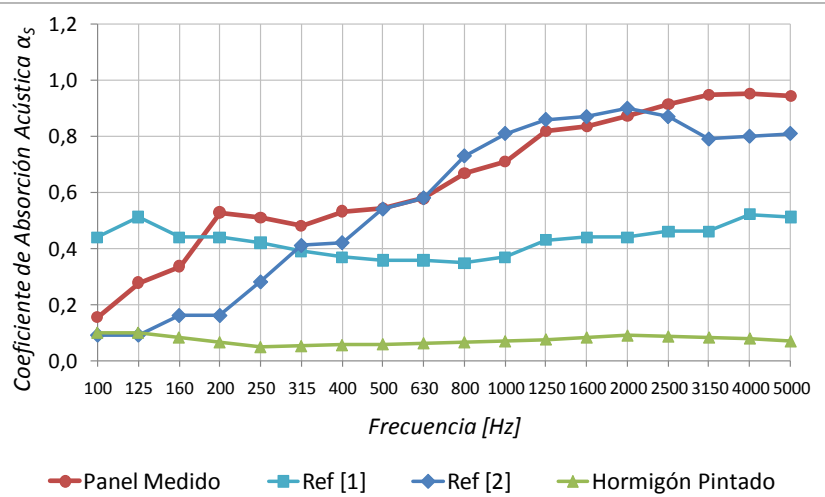

Fig. 11. Comparación de la absorción acústica de los paneles medidos con condicion de humedad B, frente a otros tipos de paneles y a un muro desnudo.

\section{CONCLUSIONES}

Como primera conclusión, se pudo determinar que el coeficiente de absorción acústica del sistema estudiado es independiente de la existencia o no de especies vegetales, sustrato y condiciones de humedad. Queda en evidencia que el buen comportamiento absorbente acústico de los módulos de soporte hace despreciable el comportamiento fonoabsorbente de la vegetación utilizada en los mismos. Esto representa una 
ventaja frente a otros sistemas en donde la absorción depende de la vegetación, y por lo tanto de su desarrollo y grado de cobertura [1].

El sistema posee una buena absorción acústica, especialmente en el rango de frecuencias altas, debido a la textura del fieltro empleado para la confección de los bolsillos soporte y al recubrimiento utilizado en los paneles de PVC espumado.

Asimismo, se observa una región alrededor de los $200 \mathrm{~Hz}$ en donde la absorción presenta un leve pico, lo cual no es característico de los fonoabsorbentes porosos puros, como lo es el fieltro. Esta mejora se debe al espacio de aire de $5 \mathrm{~cm}$ comprendido entre los módulos y el muro. Este espacio de aire además de facilitar la ventilación evitando problemas de humedad, provee un mejor desempeño fonoabsorbente respecto de un sistema montado directamente sobre el muro.
Actualmente, el trabajo de investigación continúa y se proyecta medir el aislamiento a ruido aéreo del jardín vertical.

Por otra parte, se planea a futuro ampliar la cantidad de mediciones de absorción con otros tipos de sistemas, con la intención de aportar mayor cantidad de datos en un área de conocimiento en la cual éstos son relativamente escasos y a la vez necesarios.

\section{REFERENCIAS}

[1] Z. Azkorra, G. Pérez, J. Coma, L. Z. Cabeza, S. Bures, J. E. Álvaro, A. Erkoreka y M. Urrestazaru, "Evaluation of green walls as a passive acoustic insulation system for buildings", Applied Acoustics., vol. 89, pg. 46-56, Año 2015.

[2] R. Thomazelli, F Caetano y S. Bertoli, "Acoustic properties of green walls: Absorption and insulation" en Actas ICA'16, 2016, volumen 28, 015017 (2017).

[3] ISO 354/1985 Acoustics - Measurement of sound absorption in a reverberation room.

[4] ASTM C423-02a, Standard Test Method for Sound Absorption and Sound Absorption Coefficients by the Reverberation Room Method, ASTM International, West Conshohocken, PA, 2002. 